\title{
Clinico-pathological spectrum of Childhood Central Nervous System Tumors in Iraq: A Single-Institutional Study
}

\author{
Usama Al-Jumaily, ${ }^{1}$ Rasha Al-Safi, ${ }^{2}$ Sabah Al-Mosawi, ${ }^{3}$ Homam Al-Obaidy, ${ }^{4}$ and Mohammed Fawzi ${ }^{2}$
}

Department of Pediatrics, College of Medicine, Kerbalaa University, Kerbalaa, Iraq.

2Department of Pathology, College of Medicine, Kerbalaa University, Kerbalaa, Iraq.

${ }^{3}$ Department of Pediatrics, Children Teaching Hospital, Kerbalaa, Iraq.

${ }^{4}$ College of Medicine, Kerbalaa University, Kerbalaa, Iraq.

Correspondence to Usama Al-Jumaily (email: drusama2004@yahoo.com).

(Submitted: 22 April 2019 - Revised version received: 28 April 2019 - Accepted: 17 May 2019 - Published online: 26 August 2019)

\begin{abstract}
Objectives The present retrospective study analyzed the spectrum of pediatric central nervous system (CNS) tumors in a single tertiary hospital in Iraq.

Methods Data regarding frequencies of various primary CNS tumors (diagnosed according to the World Health Organization classification), in pediatric patients ( $<18$ years of age), were collected from a single tertiary care hospital in Iraq for a period of 2014-2017.

Results Fifty-four children were diagnosed with primary CNS tumors. The most common primary pediatric CNS tumors were medulloblastoma (37\%), followed by low grade gliomas (LGGs) (29.6\%), high grade gliomas (0.011\%), CNS germinoma (0.09\%), supratentorial primitive neuro-ectodermal tumors and ependymomas (0.037\% for each). Raretumors encountered wereoligodendrogliomas, choroid plexus carcinoma, and pineoblastoma (0.18\% for each). The most common LGGs tumor was pilocytic astrocytoma.

Conclusion This is the first study reporting the spectrum of CNS tumors in children in Iraq. Except for a higher frequency of CNS germinoma, the profile of other pediatric CNS tumors in Iraq is relatively similar to that reported in other countries.

Keywords astrocytoma, epidemiology, medulloblastoma, pediatric brain tumor, World Health Organization classification
\end{abstract}

\section{Introduction}

Tumors of the nervous system in childhood occupy the second most common tumor after leukemia. ${ }^{1-4}$

They comprise approximately one-third of all childhood malignancies. In the setting of intricacy of treatment, physical and neuropsychological deficits, and neuroendocrine sequelae, they are considered one of the main causes of morbidity and mortality in children. ${ }^{5,6}$ Childhood central nervous system (CNS) tumors differ from adult brain tumors with respect to the primary site of involvement, clinical behavior, early metastases, histological and biologic characteristics. Recently, with the advent of biological investigations and the evolution of molecular and genetic studies, therapeutic approaches direct toward each specific tumor type.$^{6-9}$

This mandates a thorough knowledge of the incidence and distribution of the various CNS neoplasms. Although data from the western countries regarding the epidemiology of CNS tumors are well documented, such reliable studies are scant and nascent in developing nations. Specifically, till date, there is no documented data pertinent to the profile of pediatric CNS tumors in Iraq, which is actually very fastidious because of disparity in the management of such tumors. The present study therefore attempts to profile the clinico-pathological features of pediatric CNS tumors (according to the WHO classification) at a single tertiary center in Iraq. The data has been compared with published statistics from populationand hospital-based series.

\section{Materials and Methods}

Data of primary CNS tumors of the brain and spinal cord in the pediatric age group ( $<18$ years of age) was collected from the medical records of single tertiary hospital in Iraq, Imam Hussein Cancer Center at Kerbalaa governorate that was established in January, 2014. Because of complexity of management, the necessity for multidisciplinary approach, and practical inexperience of treating children with primary CNS tumors, this center was the first in Iraq managing those children with primary CNS tumors in addition to other malignancies. Children with various neurological symptoms with a recent diagnosis of primary CNS tumors, from different parts of the country visiting or referred to the above mentioned cancer center were properly managed according to internationally adopted protocols by surgery, radiotherapy, and chemotherapy. The conducted time period was from January 2014 to December 2017 (i.e., 4 year period study). Patients with primary CNS tumors who were younger than 18 years at the time of diagnosis was conducted. All cases with secondary CNS involvement (i.e., the primary tumor is elsewhere in the body with secondary metastases to the CNS) were excluded from the study. Data were retrieved from medical records and pathology databases. Patients included in this study were diagnosed depending on the characteristic site of the primary tumor and histological characteristics categorized according to the World Health Organization classification 2007.,10

We reviewed patients' characteristics [demographics, tumor location, pathology, living site], treatment plan (chemotherapy, surgery, and radiotherapy), and outcome.

The collected data were analyzed and compared with available published data in tumor registries and hospital-based studies.

\section{Results}

\section{Patients' Characteristics}

\section{Demographics}

From January 2014 to December 2017, 54 patients $<18$ years old with primary CNS tumors were enrolled in this study. 
There were 31 males (57.4\%) and 23 females (42.6\%) with a median age of 6 years (range 1-18 years).

Regarding the age at the time of diagnosis, 23 patients were 5 years and less, 21 patients aged between 5 and 10 years, and 10 patients were above 10 years of age.

\section{Tumor sites}

Of the 54 patients, 27 (50\%) patients had a tumor at the posterior fossa/cerebellum site; the next common sites of involvement in descending order were cerebrum (eleven patients; i.e. 20.4\%), suprasellar/hypothalamic (five patients; i.e., 9.35\%), brain stem (four patients, i.e., 7.4\%), spinal cord (two patients; i.e., 3.7\%), optic nerve (one patient; i.e., 1.9\%), and pineal gland (one patient; i.e., 1.9\%) (Table 1).

\section{Pathological diagnosis}

Of the 54 patients, 23 (42.59\%) patients had embryonal tumors histology (20 patients, medulloblastoma; two patients, supratentorial primitive neuroectodermal tumor PNET, and one patient, pineoblastoma); the second most common histology was low grade glioma (LGG) comprising 31.48\% (16, pilocytic astrocytoma; one, oligodendroglioma) of all cohort group. High grade gliomas (HGGs) were the third most common tumors (six patients, $11.1 \%$ ) followed by CNS germinoma histology (five patients, 9.25\%), ependymoma (two patients, 3.7\%), and choroid plexus carcinoma (one patient $1.85 \%$ ) (Table 2).

\begin{tabular}{|c|c|c|}
\hline Site & Frequency & Percentage \\
\hline Cerebellum & 27 & 50.0 \\
\hline Cerebrum & 11 & 20.4 \\
\hline Suprasellar & 5 & 9.3 \\
\hline Brain stem & 4 & 7.4 \\
\hline Spinal cord & 3 & 5.6 \\
\hline Optic nerve & 3 & 5.6 \\
\hline Pineal gland & 1 & 1.9 \\
\hline Total & 54 & 100.0 \\
\hline
\end{tabular}

Table 2 Frequency and percentage of pediatric CNS tumors according to histological type

\begin{tabular}{lcc} 
Histology & Frequency & Percentage \\
\hline Medulloblastoma & 20 & 37.0 \\
Pilocytic astrocytoma & 16 & 29.6 \\
HGG & 6 & 11.1 \\
CNS germinoma & 5 & 9.3 \\
Supratentorial PNET & 2 & 3.7 \\
Ependymoma & 2 & 3.7 \\
Pineoblastoma & 1 & 1.9 \\
Oligodendroglioma & 1 & 1.9 \\
Choroid plexus carcinoma & 1 & 1.9 \\
Total & 54 & 100.0
\end{tabular}

PNET, primitive neuroectodermal tumors; $\mathrm{HGG}$, high grade gliomas; CNS, central nervous system
Table 3 showed the distribution of various histological subtypes according to the original site of involvement.

\section{Living place}

Thirty-four patients (i.e., 63\%) reside in urban cities; the rest of the cohort group (i.e., 20 patients, $37 \%$ ) lives in rural regions.

\section{Modalities of treatment}

Surgery. Forty-six patients were submitted to surgery including either biopsy/PR (partial resection), subtotal resection (STR), or gross total resection (GTR). For the remaining eight patients who did not undergo any kind of surgery, the site of tumor was considered inaccessible (brain stem gliomas) or detrimental (optic nerve gliomas), and the diagnosis was made depending on the radiological findings. Another one patient with choroid plexus carcinoma was not subjected to surgery because of bad clinical status and the diagnosis was also established based on the consistent radiological appearance (Table 4).

Chemotherapy. Forty-six patients received chemotherapy adopted according to the primary histology of the tumor. Well-known international chemotherapy protocols were followed (COG and SIOP CNS tumors protocols). Only eight patients were not received chemotherapy; three of them had brain stem diffuse pontine gliomas, two were having ependymoma, and three patients with pilocytic astrocytoma who underwent complete surgical resection of their primary tumors (Table 4).

Radiotherapy. Radiotherapy (either as craniospinal irradiation or focal radiotherapy) was given to 33 patients according to recommended protocol based on the histological type and site of the tumor. The remaining 21 patients did not receive radiotherapy because of the following: 13 patients with LGGs who underwent surgery other than GTR followed by chemotherapy; another three patients with LGGs who underwent complete surgical resection and were only followed clinically and radiologically; three patients with medulloblastoma who were under 3 years of age and received only chemotherapy after surgery; one patient with choroid plexus carcinoma who received only one cycle of chemotherapy and died because of tumor progression; and another one patient with germinoma who received chemotherapy and parents refused further treatment (Table 4).

Outcomes. Out of 54 cases diagnosed at our institute, There were 14 deaths (25.9\%) in our study; the highest mortality were in embryonal tumors (seven patients) and HGG (five patients) due to disease progression; one patient with choroid plexus carcinoma also died because of advanced tumor disease, and another one patient with LGG who died because of intracranial hemorrhage related to thrombocytopenia as a sequel of chemotherapy effects. Nine patients (16.7\%) abandoned treatment or lost follow-up. Moreover, 26 patients $(48.1 \%)$ have completed treatment and are on regular follow-up, and four (7.4\%) patients are currently on treatment. One patient (1.9\%) with ependymoma is still alive with disease recurrence until the date of writing this study (Table ).

\section{Discussion}

Pediatric CNS tumors accounted on an average $14.8 \%$ of total intracranial tumors (ranging from $10 \%$ to $21 \%$ ). In our study, there is a slight male preponderance (the overall male to female ratio was 1.34:1); this ratio is variable in other reported series worldwide. . $, 5,7,11-13^{-13}$ 


\begin{tabular}{|c|c|c|c|c|c|c|c|}
\hline & BS & Cerebellum & Spinal cord & Suprasellar & Cerebrum & Pineal & Optic nerve \\
\hline$M B$ & 0 & $20(100)$ & 0 & 0 & 0 & 0 & 0 \\
\hline Pilocytic astrocytoma & $1(6.3)$ & $5(31.3)$ & $3(18.8)$ & 0 & $4(25)$ & 0 & $3(18.8)$ \\
\hline CNS germinoma & 0 & 0 & 0 & $5(100)$ & 0 & 0 & 0 \\
\hline HGG & $3(50)$ & $1(16.7)$ & 0 & 0 & $2(33.3)$ & 0 & 0 \\
\hline Pineoblastoma & 0 & 0 & 0 & 0 & 0 & $1(100)$ & 0 \\
\hline Supratentorial PNET & 0 & 0 & 0 & 0 & $2(100)$ & 0 & 0 \\
\hline Ependymoma & 0 & $1(50)$ & 0 & 0 & $1(50)$ & 0 & 0 \\
\hline Oligodendroglioma & 0 & 0 & 0 & 0 & $1(100)$ & 0 & 0 \\
\hline Choroid plexus carcinoma & 0 & 0 & 0 & 0 & $1(100)$ & 0 & 0 \\
\hline Total & 4 & 27 & 3 & 5 & 11 & 1 & 3 \\
\hline
\end{tabular}

BS, brain stem gliomas; $\mathrm{MB}$, medulloblastoma; $\mathrm{HGG}$, high grade gliomas; PNET, primitive neuroectodermal tumor.

\section{Table 4 Modalities of treatment according to various histological subtypes}

\begin{tabular}{|c|c|c|}
\hline Subtype of tumor-modalities of treatment & Frequency & Percentage \\
\hline MB - Surgery, Radiotherapy, Chemotherapy & 18 & 33.3 \\
\hline Pilocytic astrocytoma—Surgery, Chemotherapy & 9 & 16.7 \\
\hline Pilocytic astrocytoma—Chemotherapy & 4 & 7.4 \\
\hline HGG—Radiotherapy & 3 & 5.6 \\
\hline HGG - Surgery, Radiotherapy, Chemotherapy & 3 & 5.6 \\
\hline Pilocytic astrocytoma—Surgery & 3 & 5.6 \\
\hline MB_-Surgery, Chemotherapy & 2 & 3.7 \\
\hline CNS germinoma—Surgery, Radiotherapy, Chemotherapy & 2 & 3.7 \\
\hline CNS germinoma—Surgery, Chemotherapy & 2 & 3.7 \\
\hline Supratentorial PNET_Surgery, Radiotherapy, Chemotherapy & 2 & 3.7 \\
\hline Ependymoma-Surgery, Radiotherapy & 2 & 3.7 \\
\hline CNS germinoma-Radiotherapy, Chemotherapy & 1 & 1.9 \\
\hline Pineoblastoma—Surgery, Radiotherapy, Chemotherapy & 1 & 1.9 \\
\hline Oligodendroglioma_-Surgery, Radiotherapy, Chemotherapy & 1 & 1.9 \\
\hline Choroid plexus carcinoma —Chemotherapy & 1 & 1.9 \\
\hline Total & 54 & 100.0 \\
\hline
\end{tabular}

MB, medulloblastoma; $\mathrm{HGG}$, high grade gliomas; PNET, primitive neuroectodermal tumor; $C N S$, central nervous system.

\begin{tabular}{|c|c|c|c|c|c|c|c|c|c|c|}
\hline Tumor & Brazil $^{2}$ & Korea $^{13}$ & Germany $^{18}$ & Canada $^{17}$ & Beijing $^{21}$ & Sweden $^{19}$ & Morocco $^{20}$ & Japan $^{22}$ & India $^{6}$ & Iraq (current study \\
\hline LGG & 33.4 & 30.4 & 42.8 & 41.1 & 36.7 & 51 & 38.8 & 35.7 & 35.8 & 31.5 \\
\hline MB and PNET & 13.9 & 19.8 & 25.7 & 15.4 & 14.6 & 17 & 28.9 & 10 & 22.4 & 40.7 \\
\hline Ependymoma & 7.4 & 8.1 & 10.4 & 7 & 5.6 & 8 & 12 & 4.8 & 9.8 & 3.7 \\
\hline GCT & 3.6 & 8.1 & NA & 3.1 & 7.9 & 1.5 & 0.9 & 14.3 & 2 & 9.3 \\
\hline Pineal tumor & NA & NA & 1.3 & 0.5 & 0.6 & 2.7 & 0.7 & 0 & 1.3 & 1.9 \\
\hline CPC & 3 & 2.2 & NA & 2.3 & 1.8 & 1.9 & NA & 0 & 1.8 & 1.9 \\
\hline $\begin{array}{l}\text { Neuronal and mixed } \\
\text { neuronal glial }\end{array}$ & 7.6 & 6.2 & 3.2 & $<2$ & 3.1 & 0 & 1.3 & 0 & 2.4 & 0 \\
\hline Craniopharyngioma & 11 & 9.2 & 4.4 & 6.8 & 18.4 & 4.6 & 6.6 & 10.5 & 10.2 & 0 \\
\hline HGG & NA & NA & NA & NA & NA & NA & NA & NA & NA & 11.1 \\
\hline Meningeal & 3 & 2.6 & 1.2 & $<2$ & 3.1 & 1.6 & 2.2 & 1.9 & 3.2 & 0 \\
\hline Nerve sheath & NA & 0.4 & NA & 3.1 & 2.8 & 1.1 & NA & 0 & 3.6 & 0 \\
\hline
\end{tabular}

LGG, low grade gliomas; MB, medulloblastomas; PNET, primitive neuroectodermal tumors; GCT, germ cell tumors; CPC, choroid plexus carcinomas; HGG, high grade gliomas; NA, no available data. 


\begin{tabular}{lcc}
\hline Table 6 Outcome of patients with CNS tumors & \\
\hline Treatment status & Frequency & Percentage \\
\hline Complete treatment with no & 26 & 48.1 \\
evidence of tumor recurrence & 14 & 25.9 \\
Death & 9 & 16.7 \\
Abandoned treatment & 4 & 7.4 \\
On treatment & 1 & 1.9 \\
Alive with disease & 54 & 100.0 \\
Total & 1 & 1.9 \\
Pineal gland & 54 & 100.0 \\
Total & & \\
\hline
\end{tabular}

About two-third of children with CNS tumors in this study were living in urban areas, which anticipate higher incidence of such tumors in civilized rather than rural regions; this may suggest an environmental impact in the etiology of such tumors. ${ }^{14,15}$

In this study, the most common brain tumors in the pediatric age group in descending order are embryonal tumors (medulloblastoma and supratentorial PNET, and pineoblastoma), LGG (pilocytic astrocytoma and oligodendroglioma), HGG, CNS germinoma, ependymoma, and choroid plexus carcinoma. Compared with other previous reported series nationwide, it was seen that there is a higher incidence of embryonal tumors in our current study. ${ }^{1,2,5-7,11-13}$

Low grade gliomas occupy the second most common encountered tumor (i.e., 31.5\%); in other reported studies, LGG were the most common CNS tumors in pediatric age group with a percentage ranging from $30.4 \%$ to $51 \%$. $6,711-13$

High grade gliomas were the third most common CNS tumors in our study, while data were lacking from most of published studies, the incidence of HGG is variable in other studies. ${ }^{1,2}$

Germ cell tumors (GCT) are the fourth most common type comprising 9.3\%. There was a higher frequency of GCT from studies of south-east Asia including Korea, China, and Japan which may suggest a genetic and environmental influence., ${ }^{2,16}$

Although ependymomas are the third most common tumors from the international-based data, they only occupy the fifth place in this current study comprising only $3.9 \%$ of the total histological types. ${ }^{1,6,7,13}$

The incidence of craniopharyngioma reported from the various published studies is variable, ranging from $4.4 \%$ to $18.4 \%$. It was the third most common CNS tumor from data reported from Korea, Brazil, and India, while figures from Canada, Germany, Sweden, and Morocco showed that it was the fourth most common pediatric CNS tumor. ${ }^{2,6,13,17-20}$

Data from Beijing and Japan, however, showed craniopharyngiomas to be the second and third commonest tumor, respectively. ${ }^{21,22}$

Our current study, and as there is no incidence of craniopharyngioma, may not reflect the accurate burden of such tumor as most of the patients may be circulated between neurosurgeons and radiation oncologists by passing pediatric oncologists; another factor is the lack of complete registration of newly diagnosed cases with local cancer registries, leading to underestimation of such diseases.

Regarding the outcome, our results is relatively similar to those reported in developing countries based on the rate of death and frequency of abandonment, and lower than that in developed countries. ${ }^{5,23-25}$

Our major obstacles that lead to low outcome were late referral to pediatric oncologist, lack of multidisciplinary teamwork, misconception of our community that cancer will eventually lead to death, poverty and financial problems that hinder the family consulting pediatric oncologist.

We emphasize on hospital-based prevalence data to form the basis for estimating the disease incidence. This data is essential to determine the required healthcare basis in the management of these diseases, and for evaluating geographical variance in their molecular and genetic outlines. As the incidence of CNS tumors is increasing in developing countries, and because of higher morbidity and mortality comparing with other pediatric malignancies, it necessitates accurate hospital-based estimation of such tumors by collecting data from all oncology centers in Iraq in an attempt to represent the profile of the entire country.

As our center is the first tertiary center managing pediatric CNS tumors in Iraq, our study may not reflect the real distribution of patients with primary CNS tumors in children. Another limitation of our study was that not all children with CNS tumors were managed by a pediatric oncologist; some of the children are managed by adult oncologists, neurosurgeons, and radiation oncologists who they focused on surgery followed by radiotherapy without awareness of detrimental effects of radiotherapy in growing brains of children. This highlights the necessity of encouraging physicians to refer every child with CNS tumor to a tertiary center under the care of pediatric oncologists; subsequently, this will facilitate and accurately estimate the incidence of cancer in developing countries like Iraq; moreover, the value of epidemiological-based data collection by hospitals will be improved. Despite of these limitations, this study may represent an initiative to launch for further collaborative studies between cancer centers in different areas in Iraq for more precise estimation of primary CNS tumors in Iraqi children, as an endeavor to assess the whole patient group.

To conclude, there is a rising global trend in the incidence of pediatric CNS tumors. The present study may form the basis for a more wide study to provide the first profile of pediatric CNS tumors in Iraq.

\section{Conflicts of interest}

None.

\section{References}

1. Lacour B, Guyot-Goubin A, Guissou S, Bellec S, Désandes E, Clavel J. Incidence of childhood cancer in France: National Children Cancer Registries, 2000-2004. Eur J Cancer Prev. 2010;19:173-181. Epub 2010/04/03

2. Rosemberg S, Fujiwara D. Epidemiology of pediatric tumors of the nervous system according to the WHO 2000 classification: a report of 1,195 cases from a single institution. Childs Nerv Syst. 2005;21:940-944.

3. Louis DN, Ohgaki H, Wiestler OD, Cavenee WK, Burger PC, Jouvet A, et al. The 2007 WHO classification of tumours of the central nervous system. Acta Neuropathol. 2007;114:97-109.

4. Ishihara H, Ohno Y, Fujii M, Hara J, Soda M. Epidemiological analysis of childhood cancer in Japan based on population-based cancer registries, 1993-2009. Jpn J Clin Onco. 2017;47:660-663.

5. Suresh SG, Srinivasan A, Scott JX, Rao SM, Chidambaram B, Chandrasekar S. Profile and outcome of pediatric brain tumors - experience from a tertiary 
care pediatric oncology unit in South India. J Pediatr Neurosci. 2017;12:237244.

6. Jain A, Sharma MC, Suri V, Kale SS, Mahapatra AK, Tatke M, et al. Spectrum of pediatric brain tumors in India: a multi-institutional study. Neurol India 2011;59:208-211.

7. Rickert CH, Paulus W. Epidemiology of central nervous system tumors in childhood and adolescence based on the new WHO classification. Childs Nerv Syst. 2001;17:503-511.

8. Diamandis P, Aldape K. World Health Organization 2016 Classification of Central Nervous System Tumors. Neurol Clin. 2018;36:439-447.

9. DeSouza RM, Jones BR, Lowis SP, Kurian KM. Pediatric medulloblastoma - update on molecular classification driving targeted therapies. Front Oncol. 2014;4:176.

10. Fuller GN, Scheithauer BW. The 2007 Revised World Health Organization (WHO) Classification of Tumours of the Central Nervous System: newly codified entities. Brain Pathol 2007;17:304-307.

11. Asirvatham JR, Deepti AN, Chyne R, Prasad MS, Chacko AG, Rajshekhar V, et al. Pediatric tumors of the central nervous system: a retrospective study of 1,043 cases from a tertiary care center in South India. Childs Nerv Syst. 2011;27:1257-1263.

12. Madhavan R, Kannabiran BP, Nithya AM, Kani J, Balasubramaniam P, Shanmugakumar S. Pediatric brain tumors: an analysis of 5 years of data from a tertiary cancer care center, India. Indian J Cancer 2016;53:562-565.

13. Suh YL, Koo H, Kim TS, Chi JG, Park SH, Khang SK, et al. Tumors of the central nervous system in Korea: a multicenter study of 3221 cases. J Neurooncol. 2002;56:251-259.

14. Gilli IO, Joaquim AF, Tedeschi H, Dos Santos Aguiar S, Morcillo AM, Ghizoni E. Factors affecting diagnosis of primary pediatric central nervous system neoplasias in a developing country. Childs Nerv Syst. 2019;35:91-96.

15. Johnson KJ, Cullen J, Barnholtz-Sloan JS, Ostrom QT, Langer CE, Turner MC, et al. Childhood brain tumor epidemiology: a brain tumor epidemiology consortium review. Cancer Epidemiol Biomarkers Prev. 2014;23:2716-2736.
16. Kang JM, Ha J, Hong EK, Ju HY, Park BK, Shin SH, et al. A nationwide, population-based epidemiologic study of childhood brain tumors in Korea, 2005-2014: a comparison with United States data. Cancer Epidemiol Biomarkers Prev. 2019;28:409-416.

17. Kaderali Z, Lamberti-Pasculli M, Rutka JT. The changing epidemiology of paediatric brain tumours: a review from the Hospital for Sick Children. Childs Nerv Syst. 2009;25:787-793.

18. Kaatsch P, Rickert CH, Kühl J, Schüz J, Michaelis J. Population-based epidemiologic data on brain tumors in German children. Cancer 2001;92:3155-3164.

19. Hjalmars U, Kulldorff M, Wahlqvist $Y$, Lannering B. Increased incidence rates but no space-time clustering of childhood astrocytoma in Sweden, 1973-1992: a population-based study of pediatric brain tumors. Cancer 1999:85:2077-2090

20. Karkouri M, Zafad S, Khattab M, Benjaafar N, El Kacemi H, Sefiani S, et al. Epidemiologic profile of pediatric brain tumors in Morocco. Childs Nerv Syst. 2010;26:1021-1027.

21. Zhou D, Zhang Y, Liu H, Luo S, Luo L, Dai K. Epidemiology of nervous system tumors in children: a survey of 1,485 cases in Beijing Tiantan Hospital from 2001 to 2005. Pediatr Neurosurg. 2008;44:97-103.

22. Makino K, Nakamura H, Yano S, Kuratsu J. Population-based epidemiological study of primary intracranial tumors in childhood. Childs Nerv Syst. 2010;26:1029-1034

23. Wagner HP, Antic V. The problem of pediatric malignancies in the developing world. Ann NY Acad Sci. 1997;824:193-204.

24. Gajjar A, Sanford RA, Heideman R, Jenkins JJ, Walter A, Li Y, et al. Lowgrade astrocytoma: a decade of experience at St. Jude Children's Research Hospital. J Clin Oncol. 1997;15:2792-2799.

25. Rutkowski S, Bode U, Deinlein F, Ottensmeier H, Warmuth-Metz M, Soerensen N, et al. Treatment of early childhood medulloblastoma by postoperative chemotherapy alone. N Engl J Med. 2005;352:978-986.

This work is licensed under a Creative Commons Attribution-NonCommercial 3.0 Unported License which allows users to read, copy, distribute and make derivative works for non-commercial purposes from the material, as long as the author of the original work is cited properly. 